OPEN ACCESS

Edited by:

Ignazio Licata,

Institute for Scientific Methodology

(ISEM), Italy

Reviewed by:

Daniel Wohlfarth

Independent Researcher, Munich,

Germany

George Jaroszkiewicz,

University of Nottingham,

United Kingdom

*Correspondence:

Cristian López

lopez.cristian1987@gmail.com

Specialty section

This article was submitted to Interdisciplinary Physics,

a section of the journal

Frontiers in Physics

Received: 13 June 2018

Accepted: 24 August 2018

Published: 19 September 2018

Citation:

López C (2018) Seeking for a Fundamental Quantum Arrow of Time:

Time Reversal and the

Symmetry-To-Reality Inference in Standard Quantum Mechanics.

Front. Phys. 6:104

doi: 10.3389/fphy.2018.00104

\section{Seeking for a Fundamental Quantum Arrow of Time: Time Reversal and the Symmetry-To-Reality Inference in Standard Quantum Mechanics}

\author{
Cristian López ${ }^{1,2 *}$ \\ ${ }^{1}$ CONICET, University of Buenos Aires, Buenos Aires, Argentina, ${ }^{2}$ University of Lausanne, Lausanne, Switzerland
}

In this paper I shall shed some doubts on a widely-held claim: standard quantum mechanics is time-reversal invariant, and thereby, blind to the direction of time. Building bridges between physics and philosophy, I shall argue that such a claim features some puzzling assumptions that are frequently overlooked in the literature. In particular, I shall first argue that the claim involves some methodological and metaphysical commitments that should be evaluated more prudently from philosophy and from physics. Second, I shall point out that the common inference that goes from symmetry to metaphysical conclusions needs some correction and refinement to be acceptable for time symmetry and the debate around the arrow of time.

\footnotetext{
Keywords: philosophy of physics, time-reversal invariance, symmetry, arrow of time, quantum mechanics, motion reversal
}

\section{INTRODUCTION}

Symmetries play an important role in fundamental physics as they are typically taken as guides to look into physical theories' structure. Roughly, a symmetry is a transformation (a map) that leaves invariant the structure of the theory, somehow encoded in its laws. For instance, if a physical law $L$ turns out to be invariant under the operation of translating the system from one point of space to another, then it is told that the space's structure assumed by the theory is isotropic ${ }^{1}$. Thereby, such an invariance teaches us something about space's properties. This reasoning has drawn philosophers' attention as a way to approach longstanding philosophical problems from a new perspective. The nature of space and time may now be addressed by digging into physical theories' symmetries.

Philosophical and scientific literature has devoted special efforts to use this rationale to stem metaphysical conclusions about the nature of time, particularly, whether or not time has a privileged direction. There are some properties that one intuitively attaches to time: a flow-like behavior, a partial order, a privileged direction, and so on. However, such properties might not have a physical correlate in the natural world. Physical theories, as long as they are meant to express what

\footnotetext{
${ }^{1}$ In the case of General Relativity, where the space-time's geometry is strongly related to mass and energy's distribution, this claim is in need of some clarification. To begin with, different solutions to Einstein's field equations might feature different space-time's geometrical structures, which connects with divergences in parameters of each particular solution of the equations. In these cases, the isotropy of space doesn't depend only on a formal symmetry but also depends on a specific collection of parameters. This is not the case in the rest of physical theories, where the space-time's structure or space's and time's structure is, at least at first glance, independent of the physical content, and of the specific configurations of the solutions of their laws.
} 
the world is like, are supposed to also tell us whether time actually instantiates those properties. Yet, there are two kinds of questions one might raise at this stage: on the one hand, one could want to know why our world (a particular evolution of fundamental dynamical laws) looks temporally asymmetric. On the other hand, one could be interested in asking whether our fundamental physical theories imply a fundamental direction of time.

The former does not imply a fundamental direction of time as long as the temporally asymmetric behavior of our world could be a result of, for example, very special initial conditions at the beginning of the universe (for instance, due to a Past Hypothesis, see [1]), and there well might be temporally-inverted physically possible worlds conforming to the same fundamental physical laws. In this case, the direction of time is a contingent property of a particular solution (or of a set of solutions) of fundamental dynamical laws. The second question is modally stronger as the very theory (by means of its fundamental dynamical laws) forbids certain evolutions as physically possible, in particular, those going backward in time. To put it differently, when asking whether a theory implies a fundamental direction of time, one asks whether such a physical theory renders solutions in both directions of time regardless particular initial or boundary conditions of its solutions. A fundamental arrow of time is encoded in the dynamical laws, and it is not only a property of a particular solution or a set of solutions of the laws.

In this paper, I will focus on the search of a fundamental arrow of time, and thereby, on an arrow of time as a property of fundamental dynamical equations. And here the concept of symmetry comes into play: the property of having a fundamental, privileged temporal direction may be formally represented in terms of a symmetry transformation. Two notions are crucial to understand the directed nature of time (or the lack of directedness) in terms of physical symmetries: that of "time reversal," as the symmetry transformation that changes the direction of time in the frame of a physical theory, and that of "time-reversal invariance," as the symmetry resulting from the former.

So a quite interesting philosophical question so as to learn about the nature of time might be posed as following: are our fundamental physical laws invariant under time reversal? There is a widely-held consensus within the philosophical and scientific community claiming that all our fundamental physical laws are actually invariant under time reversal, that is, they are unable to distinguish between the past-to-future direction of time and the future-to-past one in a fundamental sense [see [2-5] among many others. Tim [6] also mentions this "usual approach" as part of the tradition on time and physics]. What this means, from a philosophical viewpoint, is that fundamental physical theories' space-time structure is "blind" to a fundamental direction of time, just as it is "blind" to, for instance, space locations. To get thing straight, this consensus claims that structurally fundamental physical theories are unable to distinguish between both directions of time because their fundamental dynamical equations are unable to do it. This does not mean that within each theory one cannot justify a direction of time by other means, but this won't any longer be structural or fundamental ${ }^{2}$.

The core of the consensus's claim is that positing a privileged direction of time would amount to positing unnecessary surplus structure. Additionally, by adopting an epistemic Occamist rule favoring physical and metaphysical claims that dispense surplus structure, one is epistemically forced to assume the most parsimonious structure, and thereby, to conclude that a fundamental direction of time is not an objective property of the physical world according to our best current physical theories, and that the temporally asymmetric behavior of our world must be explained by other means (see [10]).

This consensus extends also to the quantum realm. Quantum mechanics is assumed to be blind to a fundamental direction of time in so far as its fundamental dynamical equation, Schrödinger equation, is invariant under time reversal. Any time asymmetry within the theory must come either from forces or interactions, or from an interpretation-dependent dynamics, as that proposed by so-called Collapse Theories ${ }^{3}$. Intrinsically, theory's time's structure is symmetric. Most textbooks offer a formal proof for this fact: quantum mechanics' main equation, Schrödinger equation in its simplest form, remains invariant under the action of changing the direction of time.

My aim in this paper is to point out that the path going from the formal fact that quantum mechanics is time-reversal invariant to the metaphysical conclusion that there is no a fundamental privileged direction of time according to the theory should be addressed much more carefully. I shall describe two different classes of concerns, both aiming at shedding some doubts on the above-mentioned consensus. To be clear, I shall not argue

${ }^{2}$ In fact, there exist many and varied ways to introduce a direction of time in some fundamental physical theories, but, in general, they are not fundamental or structural to the theory. In standard quantum mechanics, for instance, Schrödinger equation with different kinds of Hamiltonians is non-time-reversal invariant in the majority of cases, but in such cases the dynamical equation is not considered as fundamental (see [7]), that is, expressed in the simplest form. In quantum field theory, the violation of $\mathrm{CP}$ (charge conjugation and parity respectively) by neutral Kaon decays implies the violation of $\mathrm{T}$ (time symmetry) which could be a good candidate for a fundamental arrow of time. However, there are disagreements on whether this T-violation is fundamental enough to justify a fundamental arrow of time: they might be merely a de facto asymmetry (see [2]); or even there is some controversy on the very nature and scope of the CPT theorem (see $[8,9])$.

${ }^{3}$ It is worth mentioning two potential candidates in this respect. On the one hand, most physicists are quite familiarized with the "Postulate of Collapse of the Wave Function." von Neumann [11] proposed two kinds of evolutions for any quantum system: a unitary, deterministic one obeying Schrödinger's timedependent equation, and a non-unitary, stochastic one induced by measurements. This last kind of evolutions does give us a genuine time-asymmetric process one could base a direction of time on. On the other hand, a more general formulation of the Collapse of the Wave Function (independent of measurements) is that presented by Ghirardi-Rimini-Weber (GRW) interpretation of quantum mechanics. According to this interpretation, not only do quantum systems evolve conforming to Schrödinger's time-dependent equation but also to a continuous stochastic evolution that makes the quantum system "jump" to a definite value each a certain amount of time. This evolution also gives us a fundamental direction of time since quantum systems experiment these "hits" in only one temporal direction. However, as well known, there exist interpretations of quantum mechanics that go without collapse (as Many-World interpretation or Modal interpretations). Under their views, therefore, any time asymmetry lying beyond Schrödinger's equation is either spurious (as is based on dispensable, non-required structure) or non-fundamental. 
that the consensus is mistaken altogether, but that some puzzling considerations are frequently overlooked. For the one thing, when Schrödinger equation's time-reversal invariance is taken for granted (as typically stated), this involves some methodological and metaphysical commitments that should be evaluated more prudently (which shall be developed in section Interpreting Time Reversal). For another thing, I shall point out that the common inference that goes from formal premises (concerning symmetries) to metaphysical claims (concerning reality), as it stands, needs some correction and refinement to be acceptable for time symmetry (which shall be addressed in section The "Symmetry-to-Reality Inference").

As this paper intends to offer some perspectives for future research, I shall not deepen in details. My overall aim is to provide a clear structure of discussion for the problem of a fundamental arrow of time in quantum mechanics related to symmetry reasoning, showing varied philosophical and formal aspects that, I think, deserve more attention. Furthermore, the paper intends to bridge the gap between philosophy and physics with respect to what kind of knowledge can be drawn from physical theories and what philosophical commitments are at play there.

\section{SYMMETRIES, TIME SYMMETRY AND THE "SYMMETRY-TO-REALITY INFERENCE"}

The realm of symmetries is quite flourished. Not only are there different types of symmetries, but there are also different ways to sort them out. There are internal symmetries and spacetime symmetries; there are also global symmetries and local ones; some (but not all) of them form a symmetry group being associated with the theory's symmetry group; some symmetries are continuous (as spatial translations or boosts), others are discrete (as time reversal or parity); some of them can be interpreted in either passive or active terms, but others seem not to accept some of these interpretations; some symmetries can be empirically tested or observed, others cannot; some symmetries are considered to be analytic and thereby a priori, while others considered to be synthetic and a posteriori; and so on. Literature on symmetries in physics is quite plentiful and one would hardly find an all-embracing definition of "symmetry."

In general, a symmetry can be predicated of two different mathematical entities related to physical notions: symmetry as a property of a dynamical equation, standing for a physical law, and symmetry as a property of a solution, standing for an evolution of a physical law. Brading and Castellani [12] call them "symmetry of a law" and "symmetry of an object" respectively; and Castagnino and Lombardi [13], in discussing the problem of the arrow of time in physics, point out that time-reversal symmetry should be considered as a property of dynamical laws, while reversibility instead as a property of its solutions. Two remarks are in order here: first, if a dynamical law is invariant under a symmetry transformation, it does not imply that its solutions will also be symmetric. Specific parameters or special initial conditions could yield the evolution asymmetric. Second, a fundamental arrow of time (or a fundamental time asymmetry) is supposed to be somehow encoded in the first sense of symmetry, that is, symmetry as a property of dynamical equations/laws of physics. Craig Callender claims that "when asking if the universe is TRI [time-reversal invariant], we desire to know whether it is at bottom TRI" ([14], p. 333), that is, beyond any asymmetry coming from particular configurations or parameters of solutions.

I will put aside the notion of symmetry as a property of solutions/evolutions (see [10]), and I will focus on the notion of symmetry as a property of physical laws. This notion of symmetry is often sharpened in terms of invariance: "something is invariant when it is left unaltered by a given transformation" ([12], p. 1332). This "something" in modern treatments of symmetries refers to dynamical equations of motion, that is, to the laws governing the time-evolution of physical systems. Therefore, "being invariant" is a property that physical laws properly instantiate. And a symmetry transformation is taken to be an operation acting upon states and observables in the dynamical laws.

When a law is left invariant under a given transformation, it is said that such a transformation preserves the truth of the law (see for instance $[15,16]$ ). One can expand the meaning of this definition by saying that such a symmetry transformation takes a model of the law (a possible solution according to the law) as input and returns a model in which the law is still true as an output (see for instance [17]). To put it differently, the symmetry transformation maps solutions into solutions, and non-solutions into non-solutions [18]. That is what preserving the truth of the law means.

However, even though these definitions shed some light on the meaning of symmetry, they are still too rough to be systematically applied to physics. Indeed, when one says that a symmetry transformation applies properly to equations, this means that it applies to different mathematical objects that combined in differential equations represent the dynamical laws of a physical theory, such as states, observables, and operators. So, a symmetry transformation must specify how those various objects transform under it.

After having applied the transformation to these objects contained in equations, if the so transformed dynamical equation renders physical solutions, then the physical law (formally represented by the dynamical equation) is said to be invariant under that transformation. A more formal definition of invariance of a law, then, runs as follow

Let $L$ be a law represented by an equation $E\left(s, O_{i}, D_{j}\right)=0$, where s represents a state, the $\mathrm{O}_{i}$ represent observables, and the $\mathrm{D}_{j}$ represent differential operators, and let $\mathrm{S}$ be a transformation acting upon the objects involved in the equation as $\mathrm{s} \rightarrow \mathrm{s}^{S}, \mathrm{O}_{i} \rightarrow$ $\mathrm{O}_{i}^{S}$, and $\mathrm{D}_{i} \rightarrow D_{i}^{S}$. L is invariant under the transformation $\mathrm{S}$ if and only if $\mathrm{E}\left(s^{S}, O_{i}^{S}, D_{i}^{S}\right)=0$, in the sense that $S$ preserves the structure and the physical content of $L$

"Time reversal" is the name of a particular symmetry transformation that stands for an inversion of the direction of time by performing $t \rightarrow-t$. However, as said before, a proper 
definition of time reversal should spell out how objects within a dynamical equation transform under time reversal so as to see whether the law is left unaltered after applying it to them.

In general, time reversal is formally represented by a timereversal operator (that might generally be called $T$ ), which specifies how states, observables and other operators behave under it. One may change $S$ by $T$ in the aforementioned definition so as to obtain a formal definition of time-reversal invariance. Yet, in order to get a less formal approximation to time symmetry in terms of models, one may say that a

A law $L$ is time-reversal invariant ( $T$-invariant) if and only if, if $m$ is a model of $\mathrm{L}$, then the time - reversed model $\mathrm{m}^{T}=\mathrm{T}(\mathrm{m})$ exists and is also a model of $L$.

The existence of $\mathrm{m}^{T}$ is what guarantees that $\mathrm{L}$ preserves its structure and physical content because it is also a solution (possible world) of the law. Naturally, the non - existence $\mathrm{m}^{T}$ means that the dynamical law lacks the property of being $T$ invariant (thereby, it is non-time-reversal invariant).

So far I have specified how the time-reversal symmetry can be settled in the frame of any physical theory. Nonetheless, it is neither clear nor obvious why one is entitled to draw metaphysical conclusions about the nature of time from a purely formal premise. The argument that takes symmetries as guides to reality has been called "symmetry-to-reality inference" by Shamik [15]. Actually, if one wants to put a symmetry-toreality argument to work, so to obtain philosophically relevant discoveries/results/conclusions, one is in need of specifying further constraints and premises. Roughly, what one has achieved so far is that a physical law preserves its truth in the sense that it preserves its structure and its physical content when a time-reversal transformation is applied. What this means is that the property of having a direction of time (represented by only choosing either $+t$ or $-t$ ) can vary freely without any change in either the structure or the content of the law. In other words, it does not matter from a physical viewpoint if time runs forward (conventionally, $+\mathrm{t}$ ) or backward $(-\mathrm{t}$ ), since the transformation leaves all the implications of the law intact. In this sense, the property of having a fundamental direction of time is said to be variant, and hence, eliminable. One could lay out the argument as follows:

1. The physical law $L$ is complete and literally true

2. The direction of time is a variant feature in $L$

3. One is epistemically entitled to dispense variant features

C. The direction of time is unreal for $\mathrm{L}$

Premise (1) just supposes that $L$ is a true and complete law governing the physical world. Otherwise, the inference could not even get off the ground. Premise (2) rewords that a symmetry holds. Premise (3) can be taken as a very general and widelyvalued epistemic constraint that advices us not to commit to surplus structure, an Occamist-type general rule. As for (C), the scope of the conclusion should be taken carefully. To begin with, (C) claims that there is no fundamental direction of time in the sense that the dynamical laws renders solutions in both directions of time: if one takes one evolution going forward in time, one can also obtain an evolution going backward in time that is a solution of the same law as well. As mentioned before, this does not mean that a particular solution cannot define a direction of time depending on, for instance, special initial conditions: the symmetry of the law does not imply the symmetry of its solutions. However, the fact is that a time-reversal invariant law itself does not distinguish between both directions of time, and therefore, to posit a direction of time for all its solutions should be to add otiose structure to the theory. Naturally, the symmetry-to-reality inference is committed to the idea that a fundamental temporal asymmetry should come from an asymmetry at the level of the fundamental dynamical law. ${ }^{4}$

Coming back to the argument, even though at first glance the inference seems to be a good one, there is no straightforward way to justify the step from 2 and 3 to C. As Dasgupta points out, there is a missing premise that would be required to put the inference to work and to draw a metaphysically interesting insight. In other words, one needs a further premise in order to introduce some physical content in the inference, a fourth premise that specifies why variant feature are dispensable.

In the rest of the paper, I shall first argue that providing an unequivocal justification of premise (2) is a more puzzling business than usually assumed (section Interpreting Time Reversal). Next, I shall point out that it is not obvious at all what is the content that such a hidden premise should feature in the case of time-reversal invariance (section The "Symmetryto-Reality Inference"). But prior to get to those points, I shall briefly describe in which way time-reversal symmetry is usually presented in standard quantum mechanics ${ }^{5}$ in the next section.

\section{TIME REVERSAL IN STANDARD QUANTUM MECHANICS}

Most physics textbooks commonly start out by preventing us to run along the idea that time reversal merely maps $t \rightarrow-t$ [what [19] identifies with a "merely mathematical" transformation and [7] characterizes as an "improper transformation"]. First, one writes down Schrödinger's equation in its partial derivative form as

$$
H|\psi\rangle=i \hbar \frac{\partial|\psi\rangle}{\partial t}
$$

\footnotetext{
${ }^{4}$ To appeal to non-time-reversal invariant laws to ground an arrow of time in physics could seem excessive and unnecessary. One could be just interested in explaining why our world (a particular evolution of the fundamental laws) looks temporally asymmetric, and easily to rely on particular configurations or conditions of our world. However, such a direction of time would be a contingent property and not a necessary one, and thereby, it wouldn't be a fundamental arrow of time. To be clear: non-time-reversal invariant laws imply that there is no solutions going backward in time, regardless contingent conditions or parameters of each particular solution. The direction of time would then be a necessary property of our world, because there is no possible world (an alternative solution) where the direction of time is inverted.

5 "Standard quantum mechanics" already refers to a physical theory, thus, it is already partially interpreted. I'll understand by "standard quantum mechanics" the formal machinery of the theory (Schrödinger equation and Born's Rule) plus some basic assumptions as, for instance, what kind of states are kinematically and dynamically allowed.
} 
Next, a time-reversal operator $(T)$ is defined not just as flipping the direction of time by doing $t \rightarrow-t$

$$
T H|\psi\rangle=i \hbar \frac{\partial T|\psi\rangle}{\partial T t}=H|\psi\rangle=-i \hbar \frac{\partial|\psi\rangle}{\partial t}
$$

but also taking the complex conjugate (which is at the same time an anti-unitary and anti-linear operator which I will call $K$ or following the standard) on (2).

$$
K H|\psi\rangle=-i \hbar \frac{\partial K|\psi\rangle}{\partial K t}
$$

In doing so, one finally gets an (allegedly) time-reversed Schrödinger equation, wherein $H=H^{*}$

$$
H\left|\psi^{*}\right\rangle=i \hbar \frac{\partial\left|\psi^{*}\right\rangle}{\partial t}
$$

Note that this quantum-mechanical time-reversal operator is no longer a mapping from $t$ to $-t$ but it features an anti-unitary transformation as well. Strictly, the time-reversal operator $T$ in standard quantum mechanics should be better represented as $T=U K$, where $U$ is a unitary operator and $\mathrm{K}$ (anti-unitary) is $K z K^{-1}=z^{*}$.

It is worth noticing a few things here. First of all, the features of the unitary operator $U$ are a consequence of the classical conditions for motion reversal, namely, $T X=X, T P=-P$ and $\sigma=-\sigma$ ([7], p. 34), where $X$ refers to the position operator, $P$ to momentum operator, and $\sigma$ to spin. For instance, Leslie [20] while introducing time reversal transformation in quantum mechanics, says that one requires momentum to change sign under time reversal by definition ([20], p. 377-378). Such a stipulation establishes a smooth continuity between time reversal in classical mechanics and time reversal in quantum mechanics, in the sense that both theories remain time-reversal invariant and change magnitudes in a similar way.

Secondly, a so-defined time-reversal operator is clearly antilinear and anti-unitary as it takes the complex conjugation and its inverse $\left(X^{-1}\right)$ exists. There is a voluminous and sound literature showing why time reversal should meet these requirements in quantum mechanics (for a classical defense, see [7, 21]; for an up-dated one, see [22]), but I shall come back to this point later.

Finally, under this anti-unitary time-reversal operator Schrödinger equation turnouts to be time-reversal invariant. To put it differently, Schrödinger equation equally produces pairs of time-symmetric evolutions, that is, both evolutions with $+t$ and with $-t$. In light of this result, Schrödinger equation does not pick up a fundamental privileged direction of time, as mentioned above.

Eugene [21] has put forward a neat way to interpret this time-reversal transformation in quantum mechanics in terms of an involution. Even though it is not always completely clear, the motivation underlying such a way of defining time reversal in quantum mechanics to a great extent follows Wigner's interpretation. According to Wigner, time reversal is a transformation such that, when the following operations are sequentially performed, one obtains the identity:

$$
\text { time displacement by } \begin{aligned}
\Delta t & \times \text { time reversal } \\
& \times \text { time displacement by } \Delta t \\
& \times \text { time reversal }=I
\end{aligned}
$$

What an involution means is that after applying time reversal twice, one should obtain the initial state again. According to my reading of Wigner's definition of time reversal, it implies that an appropriate time-reversal operator in quantum mechanics must meet conditions (5), come what may: to be a time-reversal operator is to be an operator that generates an involution as specified above. Next, Wigner establishes that $T$ has to preserve transition probabilities as well (otherwise, an involution would be no longer possible)

$$
|\langle\psi \mid \varphi\rangle|=|\langle T \psi \mid T \phi\rangle|
$$

His famous theorem claims that $T$ must be either unitary or antiunitary. As the unitary time-reversal operator meets neither (5) nor (6), time reversal must be represented by the anti-unitary operator, $\mathrm{T}=\mathrm{UK}$. To be clear: to the extent that only $\mathrm{T}=\mathrm{UK}$ is capable of representing an involution, it is the appropriate time-reversal operator for quantum mechanics.

\section{INTERPRETING TIME REVERSAL}

In section Time Reversal in Standard Quantum Mechanics, I showed how time reversal is typically introduced in the quantum realm. That its main dynamical equation, Schrödinger equation, be time-reversal invariant is what underpins premise (2) in the aforementioned inference. Despite this being a well-seated and widely-held position in the field of standard quantum mechanics, I would like to raise some critical remarks on such a view that partially concern some longstanding philosophical debates on the nature of time. In particular, I shall show that a latent tension between a relationalist view of time and a substantivalist one lies behind the definition of time reversal in standard quantum mechanics. In exposing this tension, some general considerations about the proper representation and the status of time-reversal invariance in quantum theories are brought to light.

The point I want to make first is that, typically, the definition of time reversal in quantum mechanics assumes a relationalist view of time. For instance, Gibson and Pollard [23] say:

\footnotetext{
"In this approach we see that no metaphysical notion of reversal of the direction of the flow of time is involved. We are led to consider time reversed processes but not reversal time itself. Although motion reversal and motion reversal invariance would be better names, we shall adhere to the accepted, if imprecise, usage" (1976: 177, italics mine.)
}

Similar claims can be found throughout the literature (see [20], p. 377 or [24], p. 266). Wigner himself states that the time-reversal operator he introduces should actually be called "motion-reversal operator" ([21], p. 325). Hence, the so-defined 
time-reversal operator is at the same time the motion-reversal operator as the former was defined as an involution.

However, this approach to time reversal is a bit vague to the extent that it is not fully clear what it is really arguing for. As far as I can see, the claim can be read in two quite different ways: (a) the approach is committed to the metaphysical idea that time is nothing but motion, and thereby, that reversing the direction of time is nothing but reversing the direction of motion; or (b) that the anti-unitary time-reversal operator fairly stands for a reversion of the direction of motion, but there is also a way to formally represent the reversion of time itself (what Gibson and Pollard call "the metaphysical notion of reversal of the direction of time"). Option (a) implies a relationalist-eliminativist view of time in so far as all there is are physical systems moving around and standing in various temporal relations; hence, reversing the direction of time can only mean reversing such temporal relation for the reason that there is nothing else to be reversed.

In turn, option (b) paves the way not only to a substantivalist view of time, but also to an alternative formal representation of time reversal. Substantivalism is the stance that time is 'something' independent from and prior to change. The structure of time is fixed absolutely, irrespective of changes in the world, and thus the structure of change supervenes on the structure of time. Thus, if an anti-unitary time-reversal operator actually stands for a reversion of the direction of motion, and it still makes sense to speak of time as something non-reducible to motion, then the question of how an inversion of the direction of time itself can be formally represented remains unanswered yet. Rephrasing, even though one can defend that a reversion of the direction of motion must be formally represented as specified in section Time Reversal in Standard Quantum Mechanics, if one does not endorse a relationalist-eliminativist view of time in addition, one is allowed to seek for another formal representation of the reversion of the direction of time itself.

The question that arises here is what such an alternative formal representation of time reversal would be. Some authors (see [1, 25], and, partially, [26]) have argued that time reversal should be represented merely by turning time's sign around, $t \rightarrow-t$. How other magnitudes behave under it should supervene on changing $t$ by $t$. Interestingly, under so defined unitary time - reversal operator, Schrodinger equation is non - time - reversal invariant in as much as it leads to it $H=$ $-i T_{H T}^{-1}$ and thus to $T H T^{-1}=-H$. But, according to the standard approach to quantum mechanics, the Hamiltonian cannot feature negative energies (technically, it must not be unbounded from below), so Schrödinger equation renders, by this added constrain to the pure formalism, no quantum mechanical solution when time is so inverted. The point is that negative energies are considered as "unphysical" by the standard approach to quantum mechanics. If a transformation turns solutions (for instance, a Hamiltonian within the positive energy spectrum) into non-solutions (a Hamiltonian featuring negative energies), then the dynamical equation is said to be non-invariant under such transformation. This is precisely the case when time reversal is defined in terms of a unitary operator.
Such a unitary time-reversal operator is typically dismissed in the literature because it changes Hamiltonian's sign, and thereby, leads to non-solutions of Schrödinger equation. At first glance, this would not be an issue: theory's dynamics simply fails to be invariant under T. However, I think that the issue is deeper than usually thought. First, one might straightforwardly dismiss the unitary representation of time reversal by taking option (a). Wigner's approach to time reversal in quantum mechanics is quite clear in that respect. In so far as the very definition of time reversal presupposes that the system must be taken back to its initial state, that is, that the second time translation in (5) exists, the anti-unitary representation of time reversal is the only way to meet that requirement. The underlying conceptual basis for this argument is however the metaphysical commitments with respect to time: time is nothing but motion, and reversing the direction of time is nothing but reversing the direction of motion. This is the hidden (metaphysical) premise that, I think, should be made explicit in the argumentation for supporting that time reversal must be formally represented by an anti-unitary operator.

The second point I would like to introduce minds the status of time symmetry. The anti-unitary time-reversal operator described in section Time Reversal in Standard Quantum Mechanics is usually said to be a theory-relative operator, which commonly means that the specific properties of the time-reversal operator have to be worked out within the framework of a physical theory. This is fully clear as it is said that time reversal must not be defined in quantum mechanics the same way it is usually defined in classical mechanics. According to this view, there is nothing like a general formal definition of time reversal applicable to all theories in fundamental physics. Hence, it is not clear at all whether there is a way to univocally link our concept of time reversal with a formal representation thereof. One can use the notion of "time reversal" abstractly and generally only as a shortcut to refer to a bunch of concrete instances of time-reversal transformations which must be, nevertheless, spelled out within a specific theoretical context. The tenet guiding this account is that any meaningful definition of time reversal cannot be conducted in isolation from a theory's particular details.

Interestingly, some authors have argued that a theory-relative time-reversal operator aims precisely at keeping the theory timereversal invariant (see [27, 28] for instance). Overall, the strategy runs as follows: assume first that the theory is time-reversal invariant, and then figure out the properties that the timereversal operator should meet so as to satisfy such a symmetry. This demand certainly relates to a more general approach to symmetries in physics that takes symmetries as 'guides to theory construction' (see [12]). Symmetries are non-contingent properties of theory's dynamics but they are rather constraints for theory's dynamics. In this sense, time-reversal symmetry would be an a priori symmetry that any worthy dynamics should meet. Naturally, if this is the strategy underlying the definition of the time-reversal operator in quantum mechanics, then the operator can only be anti-unitary.

Third, things get less clear as one takes option (b) above. On the one hand, the substantivalist is not compelled to define 
time reversal in terms of an involution, because such a definition represents motion reversal, and she means to refer to something other than motion. On the other hand, any alternative unitary representation of time reversal seems to lead us to accept that the standard quantum mechanics, even regarding the simplest Hamiltonian, is non-time-reversal invariant. At first glance, this is scandalously problematic as goes against the normal assumptions in the field (i.e., the consensus, I mentioned above): the theory lacks a symmetry that it previously had. However, once things are got straight from taking option (b), I do not see reasons to be alarmed. Where option (a) sees one symmetry (motionreversal invariance $=$ time-reversal invariance), option (b) sees two (motion-reversal invariance and time-reversal invariance as different symmetries). That the theory be non-time-reversal invariant (because, say, one chooses to represent a reversion of the direction of time itself by means of a unitary operator) does not imply that the theory is no longer motion-reversal invariant or that motion reversal must be represented differently. What is at stake by taking option (b) is the representation and status of time reversal qua an inversion of the direction of time itself, which is unavailable in option (a), as something different that the representation and status of motion reversal. But nothing implies that one should modify any aspect of motion reversal or decline motion-reversal invariance. The theory remains invariant under the anti-unitary motion-reversal operator as before.

I would like to make three further remarks regarding the points discussed in this section and the possibility of drawing metaphysical conclusions from them. Firstly, that quantum reality is objectively timeless (that is, as mentioned before, that lacks a fundamental arrow of time encoded in its formalism) in the sense that the physical law governing at that scale is time-reversal invariant naturally and straightforwardly follows from assuming a relationalist-eliminativist view of time. This is the metaphysical background that compels us to define time reversal as done in section Time reversal in standard quantum mechanics, that is, in terms of motion reversal. If the question about a fundamental arrow of time in physics is "whether this dynamical equation is time-reversal invariant", then Schrödinger equation would be interestingly time-reversal invariant provided that one assumes such a metaphysical commitment and not impose time-reversal invariance beforehand: time reversal is motion reversal, and anti-unitary $T$ is the only candidate to fairly represent the notion. What I mean by "interestingly" here is in a non-question-begging sense, as it happens when an operator is designed so as to yield a previously-decided result. Related to this last point, even though symmetries as guides to theory construction is an approach that conveys many advantages relative to analyzing and addressing symmetries, it has to be acknowledged that some philosophical problems seem to be posit in a way that is at odds with such an approach. And this might be the case with the problem of the arrow of time as introduced before. If the question of the arrow of time is "whether this dynamical equation is timereversal invariant", there is no meaningful way of answering it in terms of an approach that whereby it is previously assumed that Schrödinger equation is time-reversal invariant.
This does not mean that the fact that Schrödinger equation is time-reversal invariant in this way is not interesting at all; all I am suggesting is that this seems to be uninteresting for addressing specific philosophical problems, such as the problem of a fundamental arrow of time in standard quantum mechanics.

I think one gets to a crossroads at this point: either one assumes that our fundamental theories must be timereversal invariant a priori, but then one either declines drawing philosophical conclusion from time symmetry, or admits that the problem may not be formulated in those terms after all ${ }^{6}$; or one assumes that the problem is well-formulated, but that time symmetry cannot be imposed a priori, being instead a contingent property of dynamics (as [17] proposes). The latter only provides us with a philosophical basis to correctly formulate the problem of the arrow of time in terms of time reversal and time-reversal invariance.

Thirdly, taking the option (b) is the only way out for those that do not want to commit themselves to a relationalist view of time and wish to address the problem of a fundamental arrow of time in terms of time reversal. However, this choice cries out for a thorough approach to time reversal in quantum mechanics independent of motion reversal which, as far as I am aware, is still missing in the philosophical and scientific literature. Furthermore, this approach would imply substantial consequences for a fundamental arrow of time to the extent that there would be sound reasons to establish an objective direction of time encoded in a fundamental dynamical equation. Briefly, the idea is that the standard quantum mechanics does not work equally in both directions of time: while one obtains physically meaningful solutions in one of them (conventionally, the past-to-future direction), the time-reversed dynamical equation yields non-physically meaningful evolutions, or more drastically, non-quantum-mechanical solutions according to the standard approach to the theory. This objective, fundamental direction of time is independent of any particular initial or boundary conditions of its solutions. These consequences, I think, that should be studied carefully. In any case, I think that, as I argued, there are methodological and metaphysical grounds to pave the way to option (b), which is already controversial in itself.

\section{THE "SYMMETRY-TO-REALITY INFERENCE"}

Section Interpreting Time Reversal concerned the first two premises in the symmetry-to-reality inference. Ex hypothesi, Schrödinger equation was assumed to be a complete and literally true dynamical law at certain scale (premise 1), and some critical remarks were offered so as to define the timereversal transformation to set premise 2. Naturally, if Schrödinger

\footnotetext{
${ }^{6}$ Regarding the latter, one could argue that it is pointless to search for a fundamental arrow of time as an asymmetry of dynamical equations; instead, one should better focus on a non-fundamental direction of time as an asymmetry of solutions of the dynamical equations of a theory. See, for instance, Sachs 1987 and Wohlfarth 2017.
} 
equation fails to be time-reversal invariant, then the symmetryto-reality inference does not run as established. For the sake of the argument, let us suppose now that Schrödinger equation is actually time-reversal invariant. What this means is that the direction of time is a variant feature in Schrödinger equation. What metaphysical lesson could be drawn from this fact? As previously mentioned, one further premise (4) is required in order to obtain the conclusion of the dispensability or elimination of a privileged direction of time from the fact that the direction of time is a variant feature in Schrödinger equation. Along this last section, I shall address possible candidates for premise (4).

Various criteria to fill this extra-premise (4) can be found in philosophical discussion about symmetries. David [16] has claimed that variant features are non-difference makers, in the sense that they do not introduce any "difference in how the state of a (physically possible) world evolves in time" ([16], p. 1,159). As variant features are not different makers, and the Occamist premises advices us to dispense non-difference maker because they are redundant, one is allowed to conclude that variant features are unreal. Since the direction of time is a variant feature, one is entitled to get rid of it. However, Baker's claim should be taken carefully since it is not true that a variant feature in a dynamical law is a non-difference maker in all its solutions (physically possible worlds according to the law): there might be solutions of the dynamical law where the variant feature is still a difference maker in that solution.

Dasgupta [15] rather takes a purely epistemic criterion to fulfill premise (4). He relies on the notion of "detectability": a variant feature is undetectable as it is impossible for it to have a direct or indirect impact in our senses. Overall, the idea is that positing undetectable structure is an epistemic vice, in the sense that, everything else being equal (or near enough), one should prefer theories that do not posit undetectable structure. In respect of the direction of time, if this is a variant feature, then it follows that assuming a fundamental direction of time implies positing undetectable structure in the specified sense. This criterion is quite close to that of empirical equivalence: a symmetry leads to make true the same observation sentences, saving the same phenomena (see [29]).

John [17] and Jill [26] in turn adopt a "methodological principle of adequacy," as I shall call it. Roughly, the idea is that a physical theory should not posit more (space-time) structure than that required for its dynamics, that is, than "needed to support the laws" ([17],p. 46). If a theory's fundamental laws satisfy certain symmetries, then, by means of this methodological principle of adequacy, the space-time structure assumed by the theory and required for the dynamics must have a structure conforming to the dynamics' symmetries. If the direction of time is a variant feature in Schrödinger equation, then the space-time structure of standard quantum mechanics does not require a fundamental direction of time; otherwise, surplus structure is being unjustifiably posited. As one should go with the least structure, then it follows that the theory lacks a fundamental direction of time. To put it differently, any temporal asymmetry should come from non-structural features of the theory.
All these attempts to strengthen the symmetry-to-reality inference seek for avoiding to draw claims about reality from purely mathematical facts. They moreover allow the inference to run smoothly from premises (1), (2), and (3) to the conclusion. These criteria have in general been applied to shed some light on continuous space-time symmetries in classical contexts: for instance, the "undetectability" criterion works well for explaining why one should not commit to absolute velocities in classical mechanics (see [30]), or why a relationalist classical mechanics without absolute space can manage phenomena equally well than a classical mechanics with absolute space [see [17] for discussion]. However, it is not clear to me if they are equally useful criteria to shed some light on time-reversal symmetry, where one is virtually left clueless with respect to temporally-transformed scenarios.

To begin with, some of these criteria heavily rely on our experience and how experiments can be run in the physical world. One can translate a physical system throughout the space or boosts velocities in order to have some empirical basis for the alleged symmetry [see [31] for an analysis of symmetries and experience]. However, our experience is evidently time asymmetric. Take the detectability criterion: how could this criterion help us with time-reversal transformation? What sort of experience would be undetectable in relation to time reversal so as to take a fundamental direction of time as a formally variant feature and conclude that is thereby unreal? No experiment can be conceived in order to get some hint about time-reversal invariance on empirical basis; moreover, experience strongly suggest the contrary.

Furthermore, only dynamical equations governing highly idealized models are actually time-reversal invariant. When forces and interactions come into play, time-reversal invariance is rapidly broken ${ }^{7}$ (for discussion in classical mechanics, see $[14,32,33])$. It is not clear to what extent one should in general draw metaphysically substantial claims about reality from highly idealized models (as Hutchison argues), but a fundamental arrow of time should somehow be found there. As Craig Callender claims, "when asking if the universe is TRI [time-reversal invariance], we desire to know whether it is at bottom TRI" ([14], p. 333), meaning when all forces, interactions or initial/boundary conditions are putting aside. In any case, even though one is entitled to do the maneuver, the problem of how variant features should be interpreted still remains unsolved for time-reversal invariance. Experience-based criteria seem not to offer any help for it. The methodological principle of adequacy promoted by Earman and North might instead work well for these cases. Highly idealized models, when all forces and interaction vanish, would shed some light on the real and fundamental space-time structure of a theory. By looking into the fundamental dynamics' symmetries, one is somehow reading off space-time structure's properties from them. However, this account lacks any empirical support and is confined within pure

\footnotetext{
${ }^{7}$ Interestingly, it could be argued that this symmetry breaking might be seen as merely formal, as one gets to the highly idealized models by means of approximations and idealizations. Then, there is no real "symmetry breaking" in reality when forces and interactions are introduced, but in the idealized model.
} 
formalism, putting the whole weight on theoretical and epistemic advices.

This is not a problem per se, but it takes some risks. One has no way to devise what a temporally-mirrored world looks like. For the same reason, one has no motivation to assume that our best and fundamental physical theories should also work in such a temporally-mirrored world. If one is to read space-time structure's properties off dynamics' symmetries, going without any empirical or extra epistemic support (as detectability or empirical adequacy), then how one sets the time-reversal operator becomes more crucial, what brings us back to section Interpreting Time Reversal and the risk of being defining a timereversal operator so as to keep the space-temporal structure symmetric.

\section{FINAL REMARKS}

In this paper, I have analyzed a widely-spread claim in the philosophical and scientific literature (the "consensus") that Schrödinger equation is time-reversal invariant, and thereby, one should take standard quantum mechanics to be blind to a fundamental direction of time. As mentioned before, the claim assumes that a fundamental arrow of time, if something real, is a property of dynamical laws (and not of a particular solution), which can be expressed in terms of being invariant or not under time reversal. I have argued that this claim actually has a twofold structure: on the one hand, it is based on a particular way to set the time-reversal operator within the theory. On the other hand, the claim is mounted on the symmetry-to-reality inference.

In section Interpreting Time Reversal and section The "Symmetry-to-Reality Inference," I have raised some concerns about how this claim is structured. On the one hand, the very definition of the time-reversal operator implies some methodological and metaphysical assumptions with respect

\section{REFERENCES}

1. Albert DZ. Time and Chance. Cambridge, MA: Harvard University Press (2000).

2. Horwich P. Asymmetries in Time. Cambridge, MA: MIT Press (1987).

3. Price H. Time's Arrow and Archimedes' Point: New Directions for the Physics of Time. New York, NY: Oxford University Press (1996).

4. Zeh D. The Physical Basis of the Direction of Time. Berlin: Springer (2007).

5. Wallace D. The arrow of time in physics. In: Bardon A, Dyke H, editors. A Companion to the Philosophy of Time. West Sussex: Wiley-Blackwell (2012). p. 262-281.

6. Maudlin T. Remarks on the passing of time. Proc Aristotelian Soc. (2002) 102:237-52. doi: 10.1111/j.0066-7372.2003.00053.x

7. Sachs R. The Physics of Time Reversal. London: University Chicago Press (1987).

8. Penrose R. Road to Reality. London: Jonathan Cape (2004).

9. Greaves H. Towards a geometrical understanding of the CPT theorem. $\mathrm{Br} \mathrm{J}$ Philos Sci. (2010) 61:27-50. doi: 10.1093/bjps/axp004

10. Wohlfarth D. On the Concept of Fundamental Time Asymmetries in Physics. Veneto: Aracne editrice? (2017).

11. von Neumann J. Mathematische Grundlagen der Quantenmechanik. Berlin: Springer (1932). to the nature of time that are typically overlooked in the literature. In challenging some of those assumptions, one might also challenge the way in which the time-reversal operator must be defined, undermining the consensus. I have offered some perspectives in which those criticisms may be further developed.

On the other hand, I have evaluated the viability of the symmetry-to-reality inference for the case of time reversal. My point there was that, even though the inference could work well for certain symmetries by adding some extra premises that connects formal premises to metaphysical conclusions, it is not clear to me that the same strategy works equally well for timereversal symmetry.

The nature of time and, specifically, if there is a fundamental arrow of time in the quantum realm is a fascinating topic that steadily draws scientists' and philosophers' attention. Furthermore, some deeply-engrained conceptions about time and symmetries in physics have steered the debate to assume some claims as indisputable. This paper aimed at providing some perspectives for future research in philosophy of quantum mechanics and philosophy of time in order to put into question a widely-extended claim about time in quantum mechanics.

\section{FUNDING}

This work was supported by Grant 57919 from the John Templeton Foundation and by the National Council of Scientific and Technological Research (CONICET) from Argentina.

\section{AUTHOR CONTRIBUTIONS}

The author confirms being the sole contributor of this work and has approved it for publication.
12. Brading K, Castellani E. Symmetries and invariances in classical physics. In: Butterfield J, Earman J, editors. Handbook of the Philosophy of Science, Philosophy of Physics, Part, B. Netherlands: Elsevier (2007). p. 331-67.

13. Castagnino M, Lombardi O. The global non-entropic arrow of time: from global geometrical asymmetry to local energy flow. Synthese (2009) 169:1-25. doi: 10.1007/s11229-009-9495-y

14. Callender C. The metaphysics of time reversal: hutchison on classical mechanics. Br J Philos Sci. (1995) 46:331-40.

15. Dasgupta S. Symmetry as an epistemic notion (twice over). Br J Philos Sci. (2016) 67:837-78. doi: 10.1093/bjps/axu049

16. Baker D. Symmetry and the metaphysics of physics. Philos Compass (2010) 5:1157-1166. doi: 10.1111/j.1747-9991.2010.00361.x

17. Earman J. World Enough and Space-Time: Absolute versus Relational Theories of Space and Time. Cambridge, MA: MIT press (1989).

18. Belot G. Symmetry and equivalence. In: Batterman R, editor. The Oxford Handbook of Philosophy of Physics. Oxford: Oxford University Press (2013). p. 318-39.

19. Davies P. The Physics of Time Asymmetry. Berkeley, CA: University of California Press (1974).

20. Ballentine L. Quantum Mechanics. A modern Development. Singapur: World Scientific (1998).

21. Wigner EP. Group Theory and its Application to the Quantum Mechanics of Atomic Spectra. New York, NY: Academic Press (1959). 
22. Roberts B. Three myths about time reversal invariance in quantum theory. Philos Sci. (2017) 84:315-31. doi: 10.1086/690721

23. Gibson WM, Polard BR. Symmetry Principles in Elementary Particle Physics. Cambrige: Cambridge University Press (1976).

24. Sakurai JJ, Napolitano J. Modern Quantum Mechanics. San Francisco, CA: Adison-Wesley (2011).

25. Callender C. Is time 'Handed' in a quantum world? Proc Aristotelian Soc. (2000) 100:247-69. doi: 10.1111/j.0066-7372.2003.00015.x

26. North J. Two views on time reversal. Philos Sci. (2009) 75:201-23. doi: $10.1086 / 590199$

27. Arntzenius F, Greaves H. Time reversal in classical electromagnetism. $\mathrm{Br} J$ Philos Sci. (2009) 60:557-84. doi: 10.1093/bjps/axp015

28. Peterson D. Prospect for a new account of time reversal. Stud Hist Philos Mod Phys. (2015) 49:42-56. doi: 10.1016/j.shpsb.2015.01.001

29. Ismael J, van Fraassen B. Symmetry as guide to superfluous theoretical structure. In: Brading K, Castellani E, editors. Symmetries in Physics: Philosophical Reflections. Cambridge: Cambridge University Press (2003). p. 371-92.

30. Roberts J. A puzzle about laws, symmetries and measurability. Br J Philos Sci. (2008) 59:143-68. doi: 10.1093/bjps/axn009
31. Kosso P. The empirical status of symmetries in physics. Br J Philos Sci. (2000) 51:81-98. doi: 10.1093/bjps/51.1.81

32. Hutchison $\mathrm{K}$. Is classical mechanics really time-reversible and deterministic? Br J Philos Sci. (1993) 44:307-23. doi: 10.1093/bjps/44.2.307

33. Hutchison K. Temporal asymmetry in classical mechanics. Br J Philos Sci. (1995) 46:219-34. doi: 10.1093/bjps/46.2.219

Conflict of Interest Statement: The author declares that the research was conducted in the absence of any commercial or financial relationships that could be construed as a potential conflict of interest.

The handling editor and reviewer GJ declared their involvement as co-editors in the Research Topic, and confirm the absence of any other collaboration.

Copyright (c) 2018 López. This is an open-access article distributed under the terms of the Creative Commons Attribution License (CC BY). The use, distribution or reproduction in other forums is permitted, provided the original author(s) and the copyright owner(s) are credited and that the original publication in this journal is cited, in accordance with accepted academic practice. No use, distribution or reproduction is permitted which does not comply with these terms. 